\title{
BOUNDED ANALYTIC FUNCTIONS AND ABSOLUTELY CONTINUOUS MEASURES ${ }^{1}$
}

\section{GEORGE PIRANIAN, ALLEN L. SHIELDS AND JAMES H. WELLS}

1. Introduction. We shall use the symbol $H^{\infty}$ for the class of functions that are analytic and bounded in the unit disk $D$, and the symbol $A$ for the class of elements of $H^{\infty}$ that are continuous on the closure of $D$. For notational convenience, we shall of ten regard the unit circle $C$ and the interval $[0,2 \pi]$ as interchangeable. The purpose of this note is to prove the following theorem.

THEOREM 1. Let the sequence $\left\{a_{0}, a_{1}, \cdots\right\}$ of complex numbers have the property that for each function $\sum b_{n} z^{n}$ in $H^{\infty}$ the limit

$$
\lim _{r \rightarrow 1} \sum a_{n} b_{n} r^{n}
$$

exists and is finite. Then there exists a function $\phi \in L^{1}(0,2 \pi)$ such that

$$
a_{n}=\frac{1}{2 \pi} \int_{0}^{2 \pi} \phi(t) e^{i n t} d t=\hat{\phi}(n) \quad(n \geqq 0) .
$$

The converse is also true.

This result was conjectured by A. E. Taylor in 1951 (see [7, p. 33]). The analogous proposition for two-sided sequences $\left\{\cdots, a_{-1}, a_{0}, a_{1}, \cdots\right\}$, with the space $H^{\infty}$ of bounded analytic functions replaced by $L^{\infty}$ and with Abel convergence replaced by Cesàro convergence, had been established by $\mathrm{H}$. Steinhaus [6] in 1919.

To view the relations a little differently: if the sequence $\left\{a_{n}\right\}$ has the property described in our theorem, then it defines an additive, homogeneous functional on $H^{\infty}$. The theorem answers in the affirmative the question (raised in $[5$, p. 275]) whether this functional is continuous when the weak-star topology is imposed on $H^{\infty}$ as a subspace of $L^{\infty}$.

In $\$ \S 2$ and 3, we give a proof of Theorem 1. The crucial step is the construction of a Blaschke product whose absolute value is small on certain sets and is near to 1 (with closely controlled argument) on certain other sets. In $\S 4$, we examine Theorem 1 as a statement about multiplier transforms on certain sequence spaces.

Received by the editors July 5, 1966.

1 This work was supported by the National Science Foundation. 


\section{A Borel measure associated with $\left\{a_{n}\right\}$.}

Lемма 1. If the sequence $\left\{a_{n}\right\}$ satisfies the hypothesis in Theorem 1, then there exists a finite, complex-valued Borel measure $\mu$ on $[0,2 \pi]$ such that

$$
a_{n}=\int e^{i n t} d \mu(t)=\hat{\mu}(n) \quad(n \geqq 0) .
$$

Proof. We may assume that the sequence $\left\{a_{n}\right\}$ is bounded, since otherwise the limit (1) would be infinite for some absolutely convergent series $\sum b_{n}$. For each $r(0<r<1)$ and each element $f(z)=\sum b_{n} z^{n}$ of $A$, let

$$
\lambda_{r}(f)=\sum_{0}^{\infty} a_{n} b_{n} r^{n}
$$

Since $\left\{a_{n}\right\}$ is bounded, each $\lambda_{r}$ is a bounded linear functional on $A$, and by virtue of the Hahn-Banach theorem, we may extend it to a bounded linear functional (with the same norm) on the space of all complex-valued continuous functions on the unit circle $C$. By a theorem of F. Riesz, there exists a finite Borel measure $\mu_{r}$ such that

$$
\operatorname{Var} \mu_{r}=\left\|\lambda_{r}\right\| \quad \text { and } \quad \lambda_{r}(f)=\int f d \mu_{r} \quad(f \in A) .
$$

The hypothesis of Theorem 1 requires that for each $f$ the set $\left\{\lambda_{r}(f)\right\}$ $(0<r<1)$ is bounded. Hence, by the uniform-boundedness principle, the norms $\left\|\lambda_{r}\right\|$ are bounded. By the weak-star compactness of measures, there exists a measure $\mu$ such that

$$
\lim \int f d \mu_{r}=\int f d \mu \quad(f \in A) .
$$

To complete the proof of Lemma 1, we take successively the functions $f(z)=z^{n}(n=0,1, \cdots)$.

3. Absolute continuity of $\mu$. The absolute continuity of the measure $\mu$ constructed in $\S 2$ is a consequence of the following theorem.

Theorem 2. Let $\mu$ be a finite, complex-valued Borel measure on $|z|=1$ such that

$$
\lim _{r \rightarrow 1} \int f\left(r e^{i t}\right) d \mu(t)
$$

exists for all Blaschke products $f$. Then $\mu$ is absolutely continuous. 
REMARK 1. Theorem 2 is a generalization of the following theorem of F. and M. Riesz: A measure whose Fourier-Stieltjes coefficients vanish on one side is absolutely continuous. Indeed, if $\hat{\mu}(n)=0(n>0)$, then $\int f d \mu=0$ for all $f \in H^{\infty}$ (in particular, for all Blaschke products), and thus the limit (2) exists.

REMARK 2. Let $S_{\mu}$ denote the collection of all $f \in H^{\infty}$ for which the limit (2) exists. Clearly, $S_{\mu}$ is a vector subspace of $H^{\infty}$. Further, it is a closed set in the metric of $H^{\infty}$ (the proof of this leads to a double limit, but one of the limits is uniform over the whole open disk, not merely over compact subsets). By the hypothesis of Theorem 2, $S_{\mu}$ contains all Blaschke products. The following question (see $[4$, p. 855, Problem C]) remains open. Does there exist a proper closed vector subspace of $H^{\infty}$ that contains all Blaschke products?

REMARK 3. We do not know whether Theorem 1 remains valid if in the hypothesis we merely require that the limit (1) exists for all Blaschke products $f=\sum b_{n} z^{n}$, rather than for all $f \in H^{\infty}$.

Proof of Theorem 2. We must show that $\mu(F)=0$ for each Borel set $F$ of Lebesgue measure 0 (on $C$ or on $[0,2 \pi]$ ). By the regularity of the measure, it is enough to prove the proposition for closed sets $F$. Our proof will proceed by contradiction: we shall assume that $\mu(F) \neq 0$ for some (fixed) closed set $F$ of Lebesgue measure 0 .

We shall require several lemmas. In each lemma, we assume the hypothesis of Theorem 2. For $0<r<1$, we use the notation $f_{r}(z)$ $=f(r z)$.

LEMMA 2. Without loss of generality, we may assume that

$$
\Re{ } \mu(E) \geqq 0 \quad \text { and } \quad \Im \mu(E) \geqq 0
$$

for all Borel sets $E \subset F$.

Proof. This follows from the Jordan decomposition theorem for measures: the measure $\Re \mu$, restricted to the set $F$, is the difference of two positive measures that live on disjoint subsets of $F$; a corresponding statement applies to $\Im \mu$. Thus, if $F$ does not have the property in the lemma, we simply replace it with a subset $E$ on which each of $\Re \mu(E)$ and $\Im \mu(E)$ has constant sign. Multiplication of $\mu(E)$ with the appropriate power of $i$ then gives the desired result.

Lemma 3. The limit

$$
\lim _{r \rightarrow 1} \int_{F} f_{r} d \mu
$$

exists for every Blaschke product $f$. 
Proof. If $f$ is a Blaschke product, then

$$
\lim _{r \rightarrow 1} \int f_{r} h_{r} d \mu
$$

exists for each $h$ in the space $A$ of uniformly continuous analytic functions. This follows immediately from the hypothesis if $h$ is a power of $z$; the general case then follows from Remark 2, since the polynomials are dense in $A$.

Next we observe that $\lim \int f_{\mathrm{r}} h d \mu$ exists for each $h \in A$, since

$$
\left|\int f_{r} \cdot\left(h_{r}-h\right) d \mu\right| \leqq\|f\|\left\|h_{r}-h\right\| \operatorname{Var}(\mu) \rightarrow 0 .
$$

Next we choose a function $g \in A$ such that $g=1$ on $F$ and $|g|<1$ on the complement $F^{\prime}$ of $F$ (concerning the existence of such a function, see [2, Chapter VI, p. 81]). Then

$$
\int_{F} f_{r} d \mu=\int f_{r} g^{n} d \mu-\int_{F^{\prime}} f_{r} g^{n} d \mu .
$$

For each $n$, the first term on the right has a limit, as we have just seen; for large $n$, we can make the second term on the right arbitrarily small (uniformly in $r$ ), since

$$
\left|\int_{F^{\prime}} f_{r} g^{n} d \mu\right| \leqq\|f\| \int_{F^{\prime}}|g|^{n} d|\mu|
$$

and the right member tends to zero as $n \rightarrow \infty$, by bounded convergence.

Lemma 4. There exist closed sets $I_{p}(p=1,2, \ldots)$ such that

(i) each set $I_{p}$ is the union of a finite number $m_{p}$ of closed arcs $I_{p j}\left(j=1,2, \cdots, m_{p}\right)$, all having the same length $d_{p}$,

(ii) $m_{p} d_{p}<1 / p^{2}$,

(iii) $F=\cap I_{p}$.

Proof. Let $F_{n}$ denote the set of points whose distance from $F$ is at most $2 \pi / n$. Then

$$
F_{1} \supset F_{2} \supset \cdots \supset F, \quad \cap F_{n}=F,
$$

and therefore $\left|F_{n}\right| \rightarrow 0$ (by $|M|$ we denote the Lebesgue measure of the set $M$ ).

For each natural number $n$, we divide the unit circle into $2 n$ equal closed subarcs, and we denote by $J_{n}$ the union of the arcs that meet 
the set $F$. Then $J_{n} \subset F_{n}$, and therefore $\left|J_{n}\right| \rightarrow 0$. Finally, we extract a subsequence $\left\{J_{n_{p}}\right\}$ such that $\left|J_{n_{p}}\right|<1 / p^{2}$, and we write $I_{p}=J_{n_{p}}$.

Lemma 5. Let $r<1$ and $\epsilon>0$ be fixed. Then there exist a number $s(r<s<1)$ and a finite Blaschke product $b(z)$ whose $m$ zeros all lie on the circle $|z|=s$, such that

(i) $(1-s) m<\epsilon$,

(ii) $\left|b\left(s e^{i t}\right)\right|<\epsilon\left(e^{i t} \in F\right)$,

(iii) $|b(z)-1|<\epsilon(|z| \leqq r)$.

Proof. We define the positive number $s_{p}$ by the equation

$$
1-s_{p}^{2}=p d_{p} \text {. }
$$

Then $0<s_{p}<1$ and $s_{p} \rightarrow 1$. Let $z_{p j}$ denote the midpoint of the arc $s_{p} I_{p j}$. For each $z$ on this arc, $\left|z_{p j}-z\right|<d_{p} / 2$, and therefore

$$
\left|\left(z_{p j}-z\right) /\left(1-\bar{z}_{p j} z\right)\right| \leqq d_{p} / 2\left(1-s_{p}^{2}\right)=1 / 2 p .
$$

Hence the finite product

$$
b_{p}(z)=\prod_{1 \leqq j \leqq m_{p}} \frac{\left|z_{p j}\right|}{z_{p j}} \frac{z_{p j}-z}{1-\bar{z}_{p j} z}
$$

satisfies the inequality $\left|b_{p}(z)\right|<1 / 2 p$ for all $z \in s_{p} I_{p}$.

The number of factors of $b_{p}$ is $m_{p}$, and

$$
m_{p}\left(1-s_{p}\right)<m_{p}\left(1-s_{p}^{2}\right)=p m_{p} d_{p}<1 / p
$$

Thus the finite Blaschke product $b_{p}(z)$ satisfies (i) and (ii) for all $p>1 / \epsilon$.

To discuss (iii), we need the formula

$$
\frac{|a|}{a} \cdot \frac{a-z}{1-\bar{a} z}=1-\epsilon_{a}(z),
$$

where

$$
\epsilon_{a}(z)=(a+|a| z)(1-|a|) / a(1-\vec{a} z) .
$$

We shall assume that $1 / 2<|a|<1$. Then

$$
\left|\epsilon_{a}(z)\right| \leqq 4(1-|a|) /|1-\bar{a} z|
$$

and for $|z| \leqq r$,

$$
\left|\epsilon_{a}(z)\right| \leqq 4(1-|a|) /(1-r|a|) \leqq 4(1-|a|) /(1-r) .
$$

Now consider the finite product $b_{p}(z)$, with $p$ large enough so that $s_{p}>1 / 2$ and $s_{p}>r$. To simplify the notation, we write 


$$
b_{p}(z)=\prod\left(1-\epsilon_{a}(z)\right),
$$

where the product has $m_{p}$ factors.

For $|z| \leqq r$, we have the inequalities

(7) $\left|b_{p}(z)-1\right| \leqq \prod\left(1+\left|\epsilon_{a}(z)\right|\right)-1 \leqq\left(\exp \sum\left|\epsilon_{a}(z)\right|\right)-1$.

By (6),

$$
\sum\left|\epsilon_{a}(z)\right| \leqq 4 m_{p}\left(1-s_{p}\right) /(1-r),
$$

and therefore (3) implies that $b_{p}$ satisfies (iii) if $p$ is large enough.

Lemma 6. Suppose $b_{0}(z)$ is a finite Blaschke product, $\epsilon>0$, and $0<r<1$. Then we can find a number $s(r<s<1)$ and a finite Blaschke product $b(z)$ whose $m$ zeros all lie on the circle $|z|=s$, such that

(i) $(1-s) m<\epsilon$,

(ii) $\left|\int_{F} b_{0}\left(s e^{i t}\right) b\left(s e^{i t}\right) d \mu(t)\right|>|\mu(F)| / 24$,

(iii) $|b(z)-1|<\epsilon(|z| \leqq r)$.

Proof. We divide the unit circle into eight equal open arcs $J_{1}, \cdots, J_{8}$ whose end points all lie in the complement of $b_{0}(F)$ (this is possible, since $b_{0}(F)$ is nowhere dense). We then define the sets

$$
E_{k}=\left\{e^{i t}: b_{0}\left(e^{i t}\right) \in J_{k}\right\} \quad(k=1,2, \cdots, 8) .
$$

These sets are open and disjoint, and they cover $F$. We write $F_{k}$ $=F \cap E_{k}$. Without loss of generality, we may assume that $\left|\mu\left(F_{1}\right)\right|$ $\geqq|\mu(F)| / 8$.

We shall now use the geometrically obvious fact that if $w_{m}=r_{m} e_{m}^{\sharp t}\left(m=1,2, \cdots, n ; 0 \leqq t_{m} \leqq 3 \pi / 4\right)$, then $\left|\sum w_{m}\right| \geqq c \sum\left|w_{m}\right|$, where

$$
c=\frac{1}{2}[2-\sqrt{ } 2]^{1 / 2}>1 / 3 .
$$

Together with Lemma 2, it implies that

$$
\left|\int_{F_{1}} b_{0}\left(e^{i t}\right) d \mu(t)\right|>|\mu|\left(F_{1}\right) / 3 \geqq\left|\mu\left(F_{1}\right)\right| / 3 \geqq|\mu(F)| / 24,
$$

where $|\mu|\left(F_{1}\right)$ denotes the total variation of $\mu$ over $F_{1}$.

Let $s_{p}$ be defined as in the proof of Lemma 5. If $s_{p}$ is near enough to 1 , then

$$
\left|\int_{F_{1}} b_{0}\left(s_{p} e^{i t}\right) d \mu(t)\right|>|\mu(F)| / 24
$$

by the uniform continuity of $b_{0}(z)$.

Let $\delta=\operatorname{dist}\left(F_{1}, F \backslash F_{1}\right)$. With the notation of Lemma 4 , choose $p$ 
large enough so that $d_{p}<\delta$. In (4), let $|a|=s_{p}$ and $z=a u$, with $|u|=1$. Since $|1-u| \leqq 2\left|1-s_{p}^{2} u\right|$, it follows from (5) that

$$
\left|\epsilon_{a}(z)\right| \leqq 4\left(1-s_{p}\right) /\left|1-s_{p}^{2} u\right| \leqq 8\left(1-s_{p}\right) /|1-u| .
$$

For a fixed $p$, we select among the arcs $I_{p j}\left(j=1, \cdots, m_{p}\right)$ those that do not meet $F_{1}$ (since $d_{p}<\delta$, none of the arcs $I_{p j}$ meeting $F_{1}$ meets any of the other sets $F_{k}$ ).

We denote the midpoints of the selected arcs by $z_{p j}$, and we form the finite Blaschke product

$$
b_{p}(z)=\prod \frac{\left|z_{p j}\right|}{z_{p j}} \frac{z_{p j}-z}{1-\bar{z}_{p j} z} .
$$

Since the product has at most $m_{p}$ factors, it satisfies condition (i) of the lemma. Just as in the proof of Lemma 5, we have the inequality

$$
\left|b_{p}(z)\right|<1 / 2 p \quad \text { for } z / s_{p} \in\left(F \backslash F_{1}\right) .
$$

If $z / s_{p} \in F_{1}$ and $a$ denotes the midpoint $z_{p j}$ of some selected interval, we can write $z=a u$, where $|u|=1$ and $|\arg u|>\delta / 2$. Therefore (9) implies that $\left|\epsilon_{a}(z)\right| \leqq c\left(1-s_{p}\right) / \delta$, for some constant $c$, and therefore

$$
\sum\left|\epsilon_{a}(z)\right| \leqq c m_{p}\left(1-s_{p}\right) / \delta .
$$

By (7) and (11) we see that if $p$ is large enough, then $b_{p}(z)$ is arbitrarily near to 1 on $s_{p} F_{1}$ and arbitrarily small on $s_{p}\left(F \backslash F_{1}\right)$; therefore, in view of (8), condition (ii) of the lemma is satisfied.

Finally, just as in the proof of Lemma 5, we can show that $b_{p}(z)$ satisfies condition (iii) if $p$ is large enough. This completes the proof of Lemma 6.

To prove Theorem 2, we use alternately Lemmas 5 and 6 to construct an infinite Blaschke product

$$
f(z)=\prod b_{n}(z)
$$

with the following properties:

(i) all the zeros of $b_{n}(z)$ lie on a circle $|z|=r_{n}$;

(ii) if $n$ is odd and $e^{i t} \in F$, then $\left|b_{n}\left(r_{n} e^{i t}\right)\right|<1 / n$;

(iii) if $n$ is even, then

$$
\left|\int_{F} f_{n}\left(r_{n} e^{i t}\right) d \mu(t)\right|>|\mu(F)| / 24,
$$

where $f_{n}=b_{1} \cdots b_{n}$;

(iv) for large values of $n$, the product $b_{n+1} b_{n+2} \cdots$ is close to 1 on the disk $|z| \leqq r_{n}$. 
Clearly, the function $f$ satisfies the inequalities

$$
\begin{aligned}
& \left|\int_{F} f\left(r_{n} e^{i t}\right) d \mu(t)\right| \leqq|\mu(F)| / n \quad(n \text { odd }), \\
& \left|\int_{F} f\left(r_{n} e^{i t}\right) d \mu(t)\right|>|\mu(F)| / 24 \quad(n \text { even }) .
\end{aligned}
$$

Since this contradicts Lemma 3, the proof of Theorem 2 is complete.

The converse part of Theorem 1 is well known, and we merely indicate a proof. If $\phi \in L^{1}$ and $f \in L^{\infty}$ (in particular, if $f \in H^{\infty}$ ), then the convolution $\phi * f$ is a continuous function. The Abel mean of the Fourier series of a continuous function converges to the function uniformly; in particular, it converges at the point $z=1$. This is precisely the assertion that the limit (1) exists.

4. Multiplier transforms. Let $X$ and $Y$ be two spaces of sequences, and let $\left\{\lambda_{n}\right\}$ be a fixed sequence.

Definition. $\left\{\lambda_{n}\right\}$ is of class $(X, Y)$ if $\left\{\lambda_{n} a_{n}\right\} \in Y$ for each $\left\{a_{n}\right\} \in X$.

Let $R$ denote the space of bounded analytic functions in $D$ whose radial limit exists on every radius. We may regard $R$ and $A$ (see $\$ 1$ ) as spaces of sequences (Taylor coefficients).

Let $L_{+}$denote the space of sequences that constitute one side of the sequence of Fourier coefficients of some in tegrable function, and let $S_{+}$ denote the space of sequences that constitute one side of the sequence of Fourier-Stieltjes coefficients of some measure. That is, let $\left\{a_{n}\right\}$ $\in L_{+}\left(\left\{a_{n}\right\} \in S_{+}\right)$if and only if there exists a function $\phi \in L$ (a measure $\mu$ ) such that

$$
a_{n}=\hat{\phi}(n) \quad\left(a_{n}=\hat{\mu}(n)\right) \quad \text { for } n \geqq 0 .
$$

From Theorem 1 we obtain the following result.

Theorem 3. $\left(H^{\infty}, R\right)=\left(H^{\infty}, A\right)=\left(S_{+}, L_{+}\right)=L_{+}$.

Proof. The equation $\left(H^{\infty}, R\right)=L_{+}$is merely a restatement of Theorem 1. Indeed, the existence of the limit (1) is precisely the existence of the radial limit along the unit interval; the existence of the limit along any other radius follows by rotation.

Clearly, $\left(H^{\infty}, A\right) \subset\left(H^{\infty}, R\right)=L_{+}$. Conversely, the convolution of a function in $L^{1}$ with a function in $L^{\infty}$ is continuous.

The identity sequence $\{1,1, \cdots\}$ is in $S_{+}$, and therefore $\left(S_{+}, L_{+}\right)$ $C L_{+}$. The reverse inclusion follows from the fact that $(S, L)=L$ for two-sided sequences (see Zygmund [8, Chapter IV, Theorem 11.10]). This completes the proof of the theorem. 
The third equation in Theorem $3,\left(S_{+}, L_{+}\right)=L_{+}$, is in some sense a dual of the second equation, $\left(H^{\infty}, A\right)=L_{+}$. As we have just seen, it is easy to prove. However, we do not know how to establish the second equation without going through Theorem 1. Perhaps this is to be expected; for although $S_{+}$is the conjugate space of the Banach space $A$, the space $L_{+}$only becomes the full dual of $H^{\infty}$ when $H^{\infty}$ is given the weak-star topology; as we mentioned in the Introduction, Theorem 1 may be regarded as a statement about weak-star continuous linear functionals on $H^{\infty}$.

In conclusion, we mention a conjecture that seems to be difficult to settle (it implies Theorem 1).

CONJECTURE. Let $\left\{\phi_{n}\right\}$ be a sequence of elements in $L$ such that $\lim \int \phi_{n} f$ exists for each $f \in H^{\infty}$. Then there exists a $\phi \in L$ such that

$$
\lim \int \phi_{n} f=\int \phi f \quad \text { for all } f \in H^{\infty} .
$$

To state the problem differently: is the quotient Banach space $L / H$ weakly sequentially complete? (This question was raised in [1, pp. 180-181].) The analogous result for $L$ instead of $L / H$ (that is, with $L^{\infty}$ instead of $H^{\infty}$ in the statement above) was proved by Steinhaus [6] (see also the proof in Zygmund [8, Chapter IV, Theorem 9.13]).

In the paper immediately following this one, Kahane [3] gives a partial affirmative answer to the conjecture. He shows that there exists a $\phi \in L$ such that the relation (13) holds for all $f \in A$. This is enough to imply Theorem 1.

\section{REFERENCES}

1. L. Brown, A. Shields and K. Zeller, On absolutely convergent exponential sums, Trans. Amer. Math. Soc. 96 (1960), 162-183.

2. K. Hoffman, Banach spaces of analytic functions, Prentice-Hall, Englewood Cliffs, N. J., 1962.

3. J.-P. Kahane, Another theorem on bounded analytic functions and absolutely continuous measures, Proc. Amer. Math. Soc. 18 (1967), 827-831.

4. Analytic function theory, (Sympos. on Analytic Function Theory, Univ. of Kentucky, 1965), Bull. Amer. Math. Soc. 71 (1965), 855-857.

5. L. A. Rubel and A. L. Shields, The space of bounded holomorphic functions on a region, Ann. Inst. Fourier (Grenoble) 16 (1966), 235-277.

6. H. Steinhaus, Additive und stetige Funktionaloperationen, Math. Z. 5 (1919), 186-221.

7. A. E. Taylor, Banach spaces of functions analytic in the unit circle. II, Studia Math. 12 (1951), 25-50.

8. A. Zygmund, Trigonometric series, 2nd ed., Vol. I, Cambridge Univ. Press, Cambridge, 1959.

The UNiversity OF Michigan AND THE UNIVERSITY OF KeNTUCKY 